Cogez, P., Lécuyer, C., Le Masson, P., and Weil, B. (2020). "Organiser la rupture continue dans un écosystème industriel : la loi de Moore comme patrimoine de création collective dans l'industrie des semi-conducteurs." Entreprises et histoire, 98, (1), pp. 152-166.

Organiser la rupture continue dans un écosystème industriel - la loi de Moore comme patrimoine de création collective dans l'industrie du semi-conducteur.

\title{
Entretien $n^{\circ}$ spécial E\&H expertise et inconnu
}

Entretien avec $P$. Cogez et $C$. Lécuyer

Auteurs : Pascal Le Masson, Benoit Weil

L'industrie du semi-conducteur présente la curieuse propriété d'être à la fois extrêmement innovante et en même temps très planifiée par la loi de Moore. Dans cet entretien, Christophe Lecuyer, professeur d'histoire des sciences et des techniques et spécialiste de I'industrie des semi-conducteurs et Patrick Cogez, responsable de programme technique chez Aeneas et ancien directeur de l'innovation et de la recherche externe de ST Microelectronics, analysent ce phénomène. Ils reviennent sur l'histoire de cette industrie et sur la façon dont la loi de Moore a été collectivement gérée, au sein de l'International Technology Roadmap for Semiconductors (ITRS), de façon à être un patrimoine de création collective.

\section{Inconnu et dynamique des connaissances dans les semi- conducteurs?}

\subsection{La loi de Moore : continuité ou rupture ?}

PLM-BW : Quand on évoque des expertises pointues en constante évolution, le monde des semi-conducteurs apparaît comme un cas particulièrement emblématique et exemplaire. La frontière du connu et de l'inconnu se déplace régulièrement avec celle du possible ou de l'impossible. Ce monde des semi-conducteurs est marqué par une dynamique des connaissances particulièrement intense. Ne présente-t-il pas la curieuse propriété d'être à la fois un monde de continuité, illustré par la loi de Moore qui rythme depuis plus d'un demisiècle le progrès des performances, et de ruptures technologiques importantes ? La loi de Moore donne l'impression d'une innovation continue pour atteindre des objectifs bien définis à l'avance - mais il semble qu'il y a eu aussi des ruptures majeures dans les semi-conducteurs. N’y aurait-il pas plutôt une rupture continue?

PC : La loi de Moore donne un objectif, qui dessine une évolution continue, génération après génération, mais elle ne dit rien sur la façon d'y arriver. On peut à cet égard considérer l'activité de I'ITRS (International Technology Roadmap for Semiconductors). L'ITRS est une organisation très originale à laquelle participaient tous les acteurs de l'industrie des semiconducteurs (industriels, fabricants d'équipements, laboratoires de recherche, ...). Les travaux de l'ITRS consistaient à dire : si on veut atteindre tel objectif fixé par la loi de Moore, voilà où il faudrait être à telle date ou telle date, par exemple en termes de propriétés intrinsèques des matériaux ou de performance d'une étape de procédé de fabrication. 
L'ITRS s'efforçait de définir pour les différents horizons temporels les ruptures nécessaires, et donc l'inconnu auquel les expertises étaient confrontées, pour atteindre les objectifs de la loi de Moore. Plus spécifiquement, elle distinguait trois types d'objectifs :

- Ceux qui pouvaient être atteints par les procédés déjà maîtrisés industriellement au moment de l'élaboration de la feuille de route, sous réserve d'une démarche d'amélioration continue ;

- Ceux pour lesquels les procédés permettant de les atteindre étaient identifiés, mais devaient faire l'objet de travaux de recherche et développement pour pouvoir passer au stade industriel ;

- Enfin, ceux pour l'atteinte desquels les procédés étaient encore à inventer.

Typiquement, d'une génération de technologie à l'autre, 70 à $80 \%$ des étapes du procédé très complexe de fabrication des semi-conducteurs pouvaient reprendre des solutions connues pour la génération précédente en les améliorant mais pour les $20 \%$ restants les solutions connues n'étaient pas suffisantes. Pour résumer on peut dire qu'à chaque génération $20 \%$ des solutions doivent être repensées et les $80 \%$ restant supposent une innovation continue.

$\mathrm{CL}$ : Si on s'intéresse à l'industrie des semi-conducteurs sur la longue durée, on peut dire qu'on a eu une période de ruptures constantes dans les architectures et les technologies des transistors de leur invention aux Bell Telephone Laboratories en 1947 jusqu'au début des années 1960. Depuis le début des années 1960, la technologie planaire s'est imposée et cette base technique a été depuis considérablement élargie.

PC : Le transistor, vu de loin, a effectivement une architecture stable depuis l'introduction des technologies planaires. Mais sur cette base partagée par l'industrie des semi-conducteurs, il y a eu des ruptures très conséquentes, que ce soit sur les matériaux constitutifs, ou les équipements de fabrication employés. Par exemple, pour le transistor, on est passé de l'oxyde de Silicium à des oxydes à haute permittivité avec une grille en métal ; on a aussi eu des ruptures très fortes dans la photolithographie avec la nécessité d'augmenter la résolution de génération en génération. Plus récemment toutefois la rupture touche aussi l'architecture du transistor avec l'arrivée du FinFET (fin field-effect transistor), du SOI (Silicon On Insulator), ou des nanofils,...

PLM-BW : Si comme le rappelle un article de ce numéro l'invention du transistor a pris des décennies et que de très nombreuses alternatives d'architectures ont été explorées, on aurait pu croire qu'une fois une architecture stabilisée et une technologie développée l'industrie des semi-conducteurs allait connaitre un développement progressif à un rythme dicté par le " potentiel de la technologie ". Or la loi de Moore va imposer un rythme d'amélioration de la performance très rapide. Rares étaient ceux qui croyaient que la miniaturisation de génération en génération pourrait se poursuivre sur une aussi longue période jusqu'à atteindre des résolutions de l'ordre de quelques nanomètres. Pour poursuivre la loi de Moore n'a-t-il pas fallu réinventer en permanence les technologies?

CL: Dans l'histoire des semi-conducteurs, il y a eu des infléchissements importants: le procédé planaire ; ensuite les technologies MOS (industrialisées pendant les années 1960) ; puis les technologies " high speed CMOS " introduites par les Japonais à la fin des années 1970. Un autre changement pendant les années 1970 a été le passage de la diffusion à 
l'implantation ionique. Plus tard, les grilles des transistors ont été réalisées avec des nouveaux matériaux, des matériaux qui n'étaient plus du silicium polycristallin. La photolithographie a aussi beaucoup changé. Mais on reste aussi avec des éléments de continuité.

PC : A l'intérieur de ces architectures, des explorations très importantes ont été nécessaires pour améliorer les performances du transistor. Par exemple, au départ peu de matériaux étaient utilisés, progressivement la liste s'est enrichie jusqu'à des situations où on mobilise presque toutes les cases du tableau de Mendeleiev. C'est l'intérêt de l'ITRS qui a joué un grand rôle pour définir et baliser cette exploration. Pour construire sa roadmap l'ITRS définissait pour les principaux paramètres, par exemple les caractéristiques électriques d'un matériau ou la longueur de grille, quel était l'objectif à atteindre à tel horizon de temps, ce dont on avait besoin pour répondre aux prochaines générations. L'ITRS précisait ensuite pour chacun de ces paramètres jusqu'à quel niveau on pouvait aller dans l'état de l'art, jusqu'où on pouvait déplacer l'état de l'art avec de l'amélioration continue et indiquait à partir de quel seuil on ne savait plus faire. Situations qui requéraient un besoin de recherche pour avoir une technologie industrielle à la date voulue. La roadmap de I'ITRS se présentait sous forme de tableaux où pour un certain nombre de paramètres, un jeu de couleur, blanc, jaune, rouge permettait de visualiser l'effort à fournir pour les différents horizons de temps. Les "murs de briques rouges " pour reprendre l'expression de l'ITRS indiquaient l'inconnu, tout ce que l'on ne savait pas faire en développant les expertises connues et maîtrisées et qui exigeait d'aller au-delà.

\section{2- La loi de Moore, une forme de patrimoine pour mieux explorer l'inconnu?}

PLM \& BW : A vous écouter, la notion de rupture apparaît mal adaptée pour rendre compte des évolutions de l'industrie des semi-conducteurs. La notion de rupture est trompeuse, elle nous piège en nous incitant à regarder comme les deux faces d'une même médaille, ce qu'on savait et ce qu'on ne savait pas faire : les conquêtes sur l'inconnu rendraient obsolètes le savoir ancien. Or ce que vous décrivez nous raconte une autre histoire. On a bien d'un côté un processus d'accumulation, de sédimentation de savoirs et d'expertises qui s'amassent, $s^{\prime}$ organisent et se transmettent par exemple autour de l'architecture stabilisée du transistor. Et de l'autre des conquêtes de l'inconnu et une puissance d'exploration qui s'exerce en s'appuyant sur les savoirs accumulés. Plutôt qu'un processus où la rupture rendrait obsolètes les savoirs anciens et conduirait à de nouvelles connaissances, on aurait à la fois un patrimoine qui s'accumule et un effort d'exploration dans des directions encore inconnues. Du coup plutôt que de se demander ce qui devient obsolète il est plus intéressant de se demander ce qui a été conquis, quels sont les inconnus qui ont été explorés et comment les continuités établies ont favorisé l'apparition de nouvelles ruptures. Le travail de l'ITRS autour de la loi de Moore apparaît comme un effort systématique pour repérer ces grands inconnus que l'industrie des semi-conducteurs va explorer et les conquêtes à mener. De sorte que c'est la stabilisation même de l'architecture du transistor qui ouvre d'autres inconnus !

$\mathrm{CL}$ : Dès le début de la technologie des semi-conducteurs, il y a eu des inconnus. Par exemple le procédé planaire a été industrialisé dès la fin des années 1950. Mais personne, y compris son inventeur, Jean Hoerni, pourtant un excellent physicien, ne comprenait ni pourquoi et ni comment il marchait. Il faudra des années d'investigation pour comprendre ce qui se passe à l'interface entre les oxydes et le cristal. 
PC : Le premier des inconnus est comment faire des motifs de plus en plus petits, car l'essence de la loi de Moore est la miniaturisation. Il génère rapidement de nouveaux inconnus: comment faire des couches de plus en plus petites avec des caractéristiques différentes ? d'où les travaux sur l'implantation pour modifier la conductivité des différentes couches ou la recherche d'oxydes avec à haute ou basse permittivité. Un des points majeurs, mais pour lequel on retrouve bien cette notion d'accumulation est la science de l'interface entre les couches. Ont réussi à s'imposer les industriels qui ont su maîtriser année après année cette science des interfaces. La nécessité de gagner en résolution de génération en génération oblige également à explorer de nouveaux inconnus en matière de photolithographie. Outre les inconnus dans le domaine de la physique fondamentale que souligne Christophe Lécuyer, Il y a là des inconnus plus " technologiques " qu'il a fallu constamment ré-explorer et les industriels ont joué un rôle majeur.

\section{3- More Moore \& More than Moore aujourd'hui ?}

PLM \& BW : Dans les dernières années, est apparue la logique du « More than Moore " pour laquelle il ne s'agit plus (seulement) de réduire la taille pour gagner en puissance, mais où d'autres dimensions de la performance comme par exemple la consommation deviennent des enjeux. Suscitent-elles de nouveaux types d'inconnus et de nouvelles expertises ? La poursuite de la logique « More Moore » ne s'est-elle pas, de son côté, heurtée à de nouveaux obstacles ?

PC : La loi de Moore est une loi monodimensionnelle, la miniaturisation assure l'augmentation de la performance. Elle permet de gagner sur plusieurs tableaux : en diminuant la dimension on peut intégrer plus de fonctions sur la même surface et comme, en première approximation, on travaille " pleine plaque " ou " plein champ » en lithographie, la miniaturisation permet de gagner en coût. On gagne aussi en termes de vitesse du transistor et en termes d'énergie, voire simplement en termes d'encombrement pour certaines applications.

Par More than Moore on entend tout ce qui n'est pas uniquement drivé par l'aspect dimensionnel: capteurs d'images, MEMS... pour lesquels la performance est très liée à I'application. Dans le cas des MEMS on avait même presque un lien " un produit " - " une technologie ». Donc on n'a plus du tout la logique de généricité. On a une loi par application... Donc c'est très différent de la logique de Moore.

Dans la loi de Moore toutefois, chaque effort pour passer d'une génération à la suivante a coûté de plus en plus cher en investissement et la course à la miniaturisation a donc conduit à sélectionner un très petit nombre de joueurs. On parle de centaines de millions de dollars pour un équipement de lithographie moderne qui ne peut être rentabilisé que sur des volumes de production énormes. Finalement c'est moins l'aspect technologique pur que l'aspect économique qui a conduit certains joueurs à arrêter la course à la miniaturisation. Inversement avec More than Moore il apparaissait possible d'innover dans d'autres directions, avec d'autres enjeux et d'autres besoins de recherche. En 2005 les industriels européens ont poussé l'ITRS à introduire cette logique « more than Moore » dans son travail.

$\mathrm{CL}$ : Aujourd'hui, il reste seulement trois industriels, Intel, Samsung et TSMC qui sont capables de faire du More Moore.

PLM \& BW : on voit donc le caractère exceptionnel, inédit de la dynamique des connaissances sur une si longue période et sur une telle échelle, avec une double logique cumulative et 
exploratoire, qui suppose donc à la fois un effort d'organisation des connaissances intensif ET un effort d'exploration et de création de nouveaux champs scientifiques et techniques. Quels sont les acteurs et les mécanismes de la dynamique du secteur?

\section{Les acteurs de l'écosystème?}

PLM-BW : le secteur des semi-conducteurs est marqué par une grande variété d'acteurs. Quels rôles ont-ils joué dans cette dynamique ? Qui a joué le rôle principal : Université et labos, les start-ups et grands industriels, ou le DoD, ou encore les communautés de programmeurs indépendants, bar camp? Finalement ne faut-il pas souligner des contributions décisives de tous ? Et aussi un aspect géographique très fort ?

$\mathrm{CL}$ : Les grandes entreprises (AT\&T, IBM, Toshiba, Hitachi) et les start-up qui sont ensuite devenues de grands acteurs comme Intel et AMD ont joué un rôle majeur et ont été au cœur de l'innovation. Les universités ne sont entrées que beaucoup plus tard dans le jeu, dans les années 1980 aux USA avec l'aide de la DARPA. Stanford et Berkeley ont en fait absorbé et intégré les technologies venues de l'univers industriel. Cela a commencé très tôt, dès la première moitié des années 1950 . Stanford, par exemple, a tiré profit de sa proximité avec Shockley Semiconductor, la première start-up de la Silicon Valley spécialisée dans les semiconducteurs, pour y envoyer Jim Gibbons, un jeune professeur assistant. Gibbons y a appris les technologies de fabrication des dispositifs en silicium. Sur la base des savoir-faire acquis chez Shockley, il a transféré ces technologies vers l'université. Son laboratoire à Stanford, le Solid State Laboratory, a travaillé notamment sur l'implantation ionique, une idée venue de Shockley. C'est le sens inverse de ce qu'on raconte d'habitude sur le transfert de technologie! On trouve un peu plus tard le même phénomène à Berkeley, qui va intégrer des technologies venues de Fairchild, de Texas Instruments et de la division microélectronique de Hewlett-Packard.

PLM \& BW : quelle est la vocation de ces labos universitaires, leur place dans l'écosystème et leurs relations avec les industriels?

$\mathrm{CL}$ : Pendant les premières années, ces laboratoires forment surtout des doctorants qui iront ensuite faire de la recherche dans l'industrie. Ce n'est que plus tard que ces labos vont être capables d'innover dans les procédés, de développer de nouveaux types de circuits, par exemple dans les télécoms, qui seront repris par l'industrie. La dynamique de fond, en tout cas dans la Silicon Valley, est initialement portée par les entreprises.

PC : Pour ma part je ne peux pas remonter aussi loin et mes connaissances du secteur démarrent à partir des années 90 . II faut bien distinguer le développement des technologies de fabrication et la conception des circuits intégrés qui sont deux domaines d'innovation aux caractéristiques très différentes. Dans le second cas, en simplifiant à l'extrême, on a juste besoin d'un ordinateur pour effectuer de la recherche. La plupart des innovations en termes de conception des circuits intégrés sont venues des laboratoires, par exemple à Berkeley le Wireless Research Center. Les chercheurs de ces laboratoires développaient de nouvelles idées pour agencer les transistors afin d'améliorer la performance des circuits ou réaliser de nouvelles fonctions. Les entreprises fabriquaient les prototypes afin que les chercheurs expérimentent leurs idées en pratique. 
Pour la technologie, l'équipement de base pour faire un dispositif à l'état de l'art est extrêmement coûteux. En Europe, par exemple, seuls deux laboratoires (I'IMEC et le LETI) peuvent encore se prévaloir de pouvoir réaliser l'ensemble du procédé de fabrication, et sans doute seulement des parties de celui-ci pour les « nœuds technologiques " les plus avancés. Donc les outils de la recherche sont essentiellement chez les industriels. Les laboratoires de recherche travaillent souvent sur la base d'échantillons fournis par des industriels afin de comprendre et d'expliquer certains phénomènes physiques critiques de fond.

CL: Une part de l'innovation dans les procédés se fait dans les entreprises qui fabriquent les équipements de production, comme Applied Materials (AM).

PC: Historiquement les entreprises du semi-conducteur fabriquaient leurs propres équipements et peu à peu s'est développée une industrie de l'équipement (AM, ASML, etc...). Par la suite, ces équipementiers sont devenus fournisseurs non seulement de l'équipement mais aussi de son usage, du procédé - ils fournissent des étapes complètes de procédé. L'ambition pour AM dans les années 90 était de savoir fournir des usines clés en main. Cela ne s'est pas fait mais c'était la vision.

CL : Les Japonais deviennent des fournisseurs de plus en plus importants d'équipements pendant les années 1980 et 1990. C'est le moment où Tokyo Electron va se développer. Tokyo Electron absorbe les savoir-faire de fabrication d'Hitachi, Toshiba et autres producteurs japonais et les intègre dans leurs équipements. Ils revendent ces savoir-faire à l'échelle mondiale.

PC: ensuite la maîtrise des équipements est revenue en Europe avec ASML pour la photolithographie et aux US avec AM pour pratiquement toutes les autres étapes de procédé.

PLM \& BW : quelle est la place des utilisateurs, ils ont peu été évoqués jusqu'ici ? On voit souvent les utilisateurs comme un moteur de l'innovation mais est-ce le cas dans les semiconducteurs ? II semble que c'est finalement largement découplé grâce à la loi de Moore et aux logiques de technologies génériques? Mais peut-être est-ce aussi le signe que les utilisateurs d'aujourd'hui ne suffisent pas pour explorer les technologies pour demain. Les utilisateurs d'aujourd'hui ne sont pas nécessairement les utilisateurs de demain et en tous cas leurs usages ne sont pas forcément représentatifs des usages de demain ! Finalement une des vertus de la loi de Moore est son caractère de " promesse " : inventer les usages de demain, attirer les utilisateurs de demain! Paradoxalement la loi de Moore permet une forme de coconstruction entre des technologues qui ne connaissent pas leurs technologies et les usagers qui ne connaissent pas leurs usages?

PC : en ce qui concerne la logique (le calcul) et le stockage (les mémoires), la généricité fonctionne. On sait que si on est capable de fournir aux utilisateurs une plus grande puissance de calcul ou un espace mémoire plus important à moindre coût cela va être accepté. Et on a des vagues d'utilisation : le militaire, l'industrie, le grand public... C'est un peu différent dans le cas de la radio (les technologies pour la RF) : il y a eu de très forts échanges entre Nokia et ST pour travailler sur des roadmaps. Il a fallu travailler sur la consommation, les bandes de fréquences, les conditions d'utilisations particulières, etc... Dans ce cas les utilisateurs ont été associés à l'orientation de l'évolution technologique car c'était moins générique. 
$\mathrm{CL}$ : L'industrie des semi-conducteurs est surtout une industrie de l'offre. Elle ouvre des nouveaux marchés en réduisant le coût des transistors et des fonctions électroniques. Elle crée aussi des nouveaux utilisateurs en concevant pour eux des dispositifs qui intègrent des puces. Cela a été longtemps une des grandes forces des entreprises de la Silicon Valley. Mais les producteurs de circuits intégrés sont aussi à l'écoute de leurs utilisateurs. Dans le cas d'Intel, ils font le tour de leurs utilisateurs, leur présentent leur roadmap, voient ce qui les intéresse. Ils ajustent leur roadmap en fonction des retours qu'ils ont des entreprises qui utiliseront leurs microprocesseurs.

PLM \&BW : Ce qui voudrait dire que la loi de Moore ne suffit pas à résumer là où va être I'industrie. Qu'est-ce que dit une roadmap à un utilisateur? II ne suffit pas de dire " on va calculer plus vite " ou " cela sera plus dense ». La roadmap doit intégrer quand même des fonctionnalités " utilisateurs " pour qu'elle instaure les moyens d'un dialogue avec les clients. Quelles sont les fonctions qui ne sont ni complètement Moore, ni complètement le cahier des charges spécifique? Comme c'est une roadmap elles doivent avoir une dimension de généricité et ne pas refléter seulement un produit futur.

$\mathrm{CL}$ : La consommation par exemple est une variable critique pour certains utilisateurs, la fiabilité aussi. Ce sont des grandeurs un peu génériques à plusieurs produits mais qui ne sont ni génériques au niveau où l'est la loi de Moore, ni spécifiques au niveau d'un cahier des charges produit.

PLM \& BW : Y a-t-il des caractéristiques spécifiques par industries ? La loi de Moore semble valable pour tous les secteurs mais reste-t-il des spécificités sectorielles ?

PC : Il continue à y avoir quelques caractéristiques spécifiques - par exemple dans le militaire ou l'aéronautique, il faut pouvoir refaire un composant à l'identique 15 ans plus tard! Le spatial exige une tenue aux radiations qui n'est pas nécessaire pour des applications grand public.

PLM \& BW : quelle est la place de la recherche aujourd'hui ?

PC: la recherche joue un rôle majeur, mais toujours avec l'inconvénient des coûts expérimentaux pour tout ce qui concerne les technologies. Dans les matériaux les laboratoires ont joué un rôle majeur, car l'élaboration de nouveaux matériaux et l'exploration de leurs propriétés et de leur comportement nécessitent des investissements qui restent à portée des laboratoires.

$\mathrm{CL}$ : C'est une recherche très dirigée. La fonction de la loi de Moore, de la NTRS et de l'ITRS, c'est justement de permettre à des grandes entreprises de diriger la recherche à l'échelle nationale, puis mondiale dans les domaines qui les intéressent. La loi de Moore et les feuilles de route dirigent non seulement la recherche dans les laboratoires, mais orientent aussi les financements publics. Depuis 1992, la DARPA et les agences américaines utilisent la loi de Moore et les roadmaps pour faire leurs choix de financements dans le domaine de la microélectronique. C'est aussi le cas des grands laboratoires nationaux comme Sandia et 
Lawrence Livermore. L'argent public finance la recherche dans les universités en fonction de la loi de Moore et des roadmaps.

PLM \& BW : Mais les résultats peuvent être utiles en dehors de l'industrie. Est-ce que les connaissances de l'humanité ont progressé grâce à cela en dehors des applications industrielles et commerciales ? Est-ce que le pilotage industriel a enfermé la recherche dans une sorte de service industriel sans impact sur le progrès des sciences ou au contraire y a-t-il eu à cette occasion un progrès plus large des connaissances ? Dans quelle mesure les patrons de labo ont-ils su jouer sur deux tableaux : entendre les besoins de l'industrie et se dire avec cela je vais pouvoir repenser complètement ce que c'est que la matière, les états condensés, revisiter ce que c'est qu'un plasma. Une capacité à lire aussi dans la loi de Moore les enjeux de la grande science. Pour l'exprimer de façon caricaturale : la loi de Moore : combien de prix Nobel ?

$\mathrm{CL}$ : Ces recherches n'ont pas conduit à des conquêtes scientifiques couronnées par le prix Nobel. Mais on peut faire de la bonne recherche sans obtenir de prix Nobel. Les connaissances sur les matériaux notamment ont énormément progressé.

PC : D'une part, aujourd'hui l'immense majorité de la recherche fondamentale ne serait pas possible sans les capacités de calcul, d'analyse de données, de mesure et de communication qui ont été apportées par cette recherche. Même dans l'archétype des sciences dites "pures » que sont les mathématiques, on voit des chercheurs utiliser la puissance de calcul fournie par l'informatique, avec les assistants de preuve. D'autre part, et c'est l'essentiel, cette industrie et ces recherches ont permis une transformation complète de la société. Elles ont complètement changé la vie quotidienne, et elles sont au cœur des solutions envisagées pour répondre aux défis sociétaux auxquels nous sommes confrontés: limiter le changement climatique et ses impacts, diminuer notre empreinte environnementale, améliorer la qualité des soins en contenant les coûts, etc... sans oublier l'exemple, d'une actualité brûlante, de permettre de rompre l'isolement de beaucoup, et de continuer, même en mode dégradé, une activité économique. Même si elle n'est pas couronnée de nombreux prix Nobel, cette recherche est extrêmement bénéfique.

\section{Organiser l'écosystème d'acteurs: I'ITRS et ses prédécesseurs continentaux?}

\section{1- Organiser un écosystème d'innovation?}

PLM \& BW 1- Après avoir discuté de la dynamique des connaissances et des inconnus, puis des acteurs, intéressons-nous maintenant à l'organisation de l'écosystème des semiconducteurs. Quelles étaient les premières organisations avant l'ITRS, organisation à l'échelle continentale aux US, au Japon et en Europe ?

CL : Le principal antécédent à l'ITRS aux US est la NTRS ( $N$ pour National). L'ITRS est en fait I'ouverture de la NTRS aux non-américains. La NTRS elle-même repose sur des expériences de roadmapping qui remontent à la fin des années 1970 et au début des années 1980. Il y a eu des roadmaps internes aux entreprises, comme chez Intel et Motorola. Pour répondre au défi Japonais les sociétés américaines ont aussi créé des nouvelles institutions, la Sematech et la 
SRC (Semiconductor Research Corporation) qui ont dès leur création développé leurs propres roadmaps. Une autre feuille de route collective a été celle du National Advisory Committee for Semiconductors (NACS), organisme créé par le Congrès américain pour réfléchir au futur de la microélectronique au moment où cette industrie connaissait une grave crise aux EtatsUnis du fait de la concurrence japonaise. La feuille de route de la NACS, Microtech, a défini toutes les étapes nécessaires pour fabriquer des mémoires extrêmement complexes sur un horizon de neuf ans. Le National Advisory Committee for Semiconductors a ensuite demandé à Gordon Moore, président du conseil d'administration d'Intel et chairman du comité stratégique du syndicat professionnel de l'industrie des semi-conducteurs, de reprendre Microtech et de la développer.

Répondant à cette requête, Gordon Moore a créé en 1991-92 une nouvelle institution, la NTRS (National Technology Roadmap for Semiconductors). Et il l'a fait en collaboration avec IBM, Motorola et Texas Instruments. Ces quatre entreprises dominaient I'industrie des semiconducteurs à cette époque. Mais dès le milieu des années 1990, Paolo Gargini, un cadre supérieur d'Intel, a pris le contrôle de la NTRS. Il l'a réorientée et l'a focalisée sur un horizon temporel plus lointain, sur ce qui allait se passer dans cinq à quinze ans. II l'a aussi poussée à définir des objectifs très précis, de façon à ce que l'exercice soit plus utile pour Intel. Plus tard, Gargini et le PDG d'Intel, Craig Barrett, ont compris que les ressources financières et techniques des États-Unis ne suffiraient pas pour résoudre les problèmes techniques qui se profilaient sur un horizon de dix ans. Ils auraient aussi besoin pour ce faire des capacités techniques et financières des Européens, Japonais, Taïwanais et Coréens. Ce constat les a poussés à ouvrir la NTRS aux entreprises étrangères en créant I'International Technology Roadmap for Semiconductors (ITRS) à la fin des années 1990. Les Japonais semblent avoir eu leur propre roadmap nationale, mais ils l'ont abandonnée et ont décidé d'entrer dans la roadmap internationale.

\section{2- L'ITRS}

PLM \& BW : avec l'ITRS on voit apparaître une notion de roadmap très originale qui serait une roadmap de l'inconnu et pas une planification des technologies comme si elles étaient connues; quel est son intérêt?

PC : cette roadmap sert vraiment à définir ce qu'on ne sait PAS faire et quand on en aura besoin. Elle a deux intérêts stratégiques majeurs pour les industriels du semi-conducteur. D'une part orienter l'ensemble de l'écosystème, à la fois les chercheurs et les équipementiers, vers ce but-là. Et d'autre part dire aux pouvoirs publics c'est cela qu'ils doivent financer en priorité.

PLM \& BW : cette capacité d'organiser de façon collaborative l'évolution de l'ensemble de l'écosystème est assez surprenante, elle devrait a priori rencontrer beaucoup d'obstacles ! on s'attend à des comportements de passagers clandestins, des logiques de leurres, des problèmes de confidentialité ?

PC : Sur l'aspect confidentialité, en fait dire qu'on ne sait pas faire n'est pas si confidentiel surtout si l'horizon temporel est lointain! Plus on se rapproche, plus c'est difficile. Pour l'anecdote : il y a eu une édition de l'ITRS, dominée par Intel, qui disait que le passage à la 
grille métallique serait à plus de deux ans et 6 mois plus tard Intel annonçait le passage à la grille métallique. Donc là c'était clairement un leurre vis-à-vis de leurs concurrents !

Je voudrais donner un autre exemple. La recherche sur le passage du $300 \mathrm{~mm}$ au $450 \mathrm{~mm}$ a été pilotée et encouragée par Intel, Samsung et TSMC - pour des raisons économiques officiellement (réduire les coûts unitaires de manufacturing) mais en réalité il fallait des volumes de vente conséquents que seuls ces trois entreprises pouvaient atteindre et donc c'était une façon d'écarter les autres concurrents. Ils ont poussé l'ensemble de la filière à aller dans cette direction. On avait des débats importants au sein de l'ITRS mais compte tenu du poids d'Intel on voyait toujours la transition vers le $450 \mathrm{~mm}$ annoncée pour un avenir beaucoup plus proche que ce que les autres acteurs croyaient possible. Et finalement on a arrêté de parler du 450mm quand le leadership d'Intel est apparu tellement fort qu'ils n'avaient même plus besoin de passer au $450 \mathrm{~mm}$ pour asseoir cette position.

$C L$ : Pour Intel, cette roadmap a été une façon de diriger les recherches et les financements publics, mais cela a aussi été aussi un mécanisme pour battre la concurrence. La feuille de route interne d'Intel a toujours été en avance par rapport à la roadmap collective.

PLM \& BW : Parmi les obstacles, il y a aussi des conditions cognitives. Comment savoir apprécier les interactions entre les percées technologiques qui n'existent pas encore ? Une voie nouvelle ne va-t-elle par avoir des conséquences très négatives sur une autre dimension?

PC : quand on regarde les éditions successives de l'ITRS, par exemple l'évolution des objectifs sur les couches d'oxyde à basse permittivité, on voit qu'on a été de moins en moins exigeant au cours du temps sur les paramètres individuels, tout en maintenant l'objectif global (la poursuite de la loi de Moore) ! Et cela s'explique par le fait que des découvertes dans d'autres domaines ont régulièrement relâché les contraintes sur le matériau. L'ITRS a d'ailleurs introduit la notion de "equivalent scaling": on continue à atteindre les objectifs de performance et moindre consommation, mais pas nécessairement en ne travaillant que sur les dimensions du transistor. Les interactions entre les groupes de travail (TWG) sont très importantes.

$\mathrm{CL}$ : Gordon Moore et ses successeurs à Intel sont de très bons ingénieurs des procédés qui s'intéressent aux interactions entre avancées techniques et pensent systématiquement au très long terme. Dès les années 1960, ils ont appris à jouer des parties d'échec dans l'inconnu.

PLM \& BW : il y aurait là une forme d'expertise un peu particulière de la roadmap de l'inconnu, cette capacité à se projeter dans des scénarios alternatifs variés, tenant compte de ce qu'on ne sait pas faire aujourd'hui mais qu'on saura peut-être faire demain, une densité de savoir qui permet de penser tout de suite les parties d'échec qu'on ne sait pas jouer. Et aussi une expertise des interactions qu'on ne connaît pas encore ? Cette capacité à raisonner à la fois plus long terme et plus fin attesterait d'une montée en exigence cognitive ?

$C L$ : La grande expertise d'Intel, c'est l'intégration des procédés.

PC : Dans le semi-conducteur les exigences sur le procédé sont extrêmes. Pousser le contrôle de procédé à un très haut niveau d'exigence est une nécessité. II faut toujours en savoir énormément, on doit contrôler le procédé de façon extrêmement fine. Et la stratégie d'Intel 
a été d'utiliser le même équipement pour apprendre et pour faire le volume, pour éviter le problème de transfert. C'est une nécessité de savoir discuter les interactions en profondeur

PLM \& BW : sur l'organisation interne de l'ITRS comment cela fonctionnait-il, qui participait ?

PC : L'organisation de l'ITRS est basée sur une quinzaine de groupes de travail, les TWG (Technology Working Group). Chaque groupe de travail a un chairman et chaque entreprise peut décider d'envoyer des experts participer. Le principe de fonctionnement est la cooptation. Un des points critiques est la présentation en séance plénière de l'avancée des différents TWG avec à ce niveau une possibilité d'identifier les interactions d'un TWG à l'autre : il y a un enjeu à pouvoir s'ajuster et prendre en compte ce que disent les autres. Au cours des années, il y a une très grande stabilité des participants. Stabilité qui joue un rôle important en termes de compétences des experts en particulier sur ce qu'est une roadmap et comment on la construit.

PLM \& BW: précisément, quelle est la nature de ces expertises ? Est-ce que ça se rapprocherait d'un collège protoépistémique (comme décrit par $\mathrm{BC}$ dans ce numéro à propos de la dynamique de l'expertise chez ST) ?

PC : c'est à la fois très stable - les gens partent peu. Mais le noyau de départ grossit, il y a un phénomène d'agrégation. De plus, le nombre des groupes de travail augmentent également, et passe de moins de 10 en 1999 (première édition internationale) à 16 lors de la dernière édition (2013).

Parmi ceux-ci, le TWG « Emerging devices and materials » est plus spécialement orienté vers l'exploration, l'identification des technologies émergentes dans les laboratoires et chez les grands industriels.

\section{3- Les entreprises dans I'ITRS - le cas de ST Microelectronics}

PLM-BW- comment une entreprise interagit avec l'ITRS ? cf. thèse de BC: la société protoépistémique d'experts fait le pont entre ITRS et dynamique des compétences internes?

PC : Tout d'abord ce n'est pas I'ITRS qui définit la stratégie de ST - I'ITRS définit vers où va I'industrie et ST se positionne par rapport à ça. Et l'ITRS a un énorme effet sur les financements publics sur la recherche et sur les stratégies des fournisseurs. ST avait un intérêt à ce que sa stratégie et les orientations de l'ITRS soient compatibles. C'est pour ça que ST avait un intérêt à ce que le More than Moore apparaisse dans I'ITRS, ainsi que la technologie SOI (Silicon on Insulator), technologie développée par ST en liaison avec SOITEC. Sur ce dernier exemple cela a donné lieu à des discussions très fortes au niveau de l'ITRS pour que le SOI n'apparaisse pas comme un épiphénomène mais soit mentionnée comme une voie possible dans la roadmap pour faire des circuits à basse consommation donc une alternative crédible au FinFet poussé par Intel.

CL : L'ITRS envoie un signal aux fournisseurs et pour le SOI c'était important.

PLM\& BW : Y a-t-il des exemples de technologies qui se sont développées sans figurer dans la roadmap de I'ITRS? 
PC : il y a aussi des points où l'ITRS ne disait rien : les capteurs d'images n'y ont jamais figuré. ST a essayé de les introduire dans la roadmap mais il y avait trop peu d'acteurs concernés. L'architecture de la " brique de base " (le pixel) était sans doute aussi encore trop instable, avec des variations selon les fabricants. Les MEMS (Micro Electro Mechanical Systems) n'ont été introduits qu'en 2005. L'ITRS est un influenceur mais ce n'est pas le seul. II y a bien des choses en dehors de l'ITRS !

CL : L'ITRS n'a pas seulement établi des objectifs sur le très long terme. Elle a aussi identifié des solutions potentielles aux problèmes qui se posaient. Dans certains cas, les experts de I'ITRS n'ont pas vu les solutions qui allaient être ensuite adoptées par l'industrie. II me semble que cela a été le cas pour la photolithographie computationnelle.

PLM \& BW : L'ITRS a-t-il été une façon d'organiser une forme d'oligopole ? On aurait pu imaginer qu'il fasse l'objet d'une réglementation européenne pour éviter la concentration dans ce secteur. Mais on peut aussi s'interroger : sans l'ITRS est ce que le rythme aurait été aussi rapide ? A quelle point l'ITRS en concentrant tous les efforts sur la miniaturisation n'a pas laissé des voies orphelines et n'a peut-être pas permis d'explorer d'autres voies pour le développement des semi-conducteurs?

PC : ça a été clairement un moteur qui a tiré la recherche et maintenu le rythme - Mais est-ce I'ITRS ou la loi de Moore?

CL : La loi de Moore existait avant l'ITRS. Mais la NTRS et l'ITRS sont devenues des formes d'organisation nécessaires pour suivre la loi de Moore. Elles ont probablement permis que la loi de Moore ne capote pas dans les années 1990. II a fallu cette concentration d'efforts d'exploration à l'échelle mondiale pour pouvoir avancer. Il est très probable que les roadmaps ont fermé des portes techniques. Les dirigeants de la NTRS en étaient d'ailleurs très conscients.

PC : I'ITRS traduisait la loi de Moore en objectifs d'exploration dans tous les labos de recherche du monde. Les thèses commençaient toutes par l' " ITRS dit que ce sujet est important et c'est sur cette question-là que je vais travailler ».

\section{4- Pourquoi la fin de l’ITRS?}

PC : L'ITRS était un outil pour Intel et Intel s'en est désengagé, au point de laisser partir de l'entreprise le Chairman de I'ITRS. Une hypothèse est qu'Intel n'avait plus besoin de cet instrument parce que l'entreprise bénéficiait d'une position suffisamment dominante pour orienter directement les équipementiers. Par exemple, Intel a mis énormément d'argent sur la table pour travailler sur l'extrême UV directement avec ASML. IBM de son côté a dit quitter la miniaturisation et a voulu travailler sur de nouvelles architectures de calcul qui ne seraient plus basées sur le transistor, donc ce qui se passait dans l'ITRS ne les intéressait plus. Les industriels européens ont constaté que l'ITRS n'intégrait pas bien le More than Moore, peutêtre faute d'une brique équivalente au transistor dans le More Moore. 
$\mathrm{CL}$ : Un de mes informateurs japonais, un ingénieur qui participait aux TWG Emerging devices et Process Integration, $\mathrm{m}^{\prime}$ a dit avoir vu des tensions apparaître entre Intel, TSMC et Samsung à partir de 2010. Ces entreprises avaient de plus en plus de mal à arriver à un consensus et leurs roadmaps internes étaient de plus en plus divergentes. Ces tensions ont sans doute conduit à l'affaiblissement, puis à la disparition de l'ITRS. La fin de I'ITRS peut aussi s'expliquer par l'évolution des chiffres d'affaire de TSMC et Samsung qui se sont de plus en plus rapprochés de celui d'Intel. En 2017, c'est Samsung qui est arrivé à la première place en matière de ventes. TSMC et Samsung, qui ont pendant longtemps suivi Intel, ne voulaient plus jouer le jeu.

PC : Néanmoins, ils étaient tous les trois sur le FinFET et avaient des intérêts communs.

$\mathrm{CL}$ : Selon cet ingénieur japonais, les divergences sont apparues surtout dans le TWG Intégration. TSMC poussait à une cadence plus élevée et Intel résistait, car ils n'arrivaient plus à soutenir le rythme.

PC: la vitesse d'évolution entre deux génération technologiques, l'accélération ou le ralentissement de la loi de Moore a pu jouer un rôle. Car à cette période Intel a commencé à avoir des problèmes technologiques. Alors qu'ils étaient auparavant en avance sur la loi de Moore, ils ont commencé à avoir du mal à suivre.

CL : Pendant cette même période, Intel s'est financiarisé. L'entreprise a racheté ses actions pour plusieurs dizaines de milliards de dollars. Cet argent n'est pas allé au financement de la recherche et au développement de nouveaux procédés. Cela a dû jouer un rôle dans les difficultés qu'Intel a rencontrées pour suivre la dynamique technique.

PLM \& BW : On retrouve dans les explications de l'effondrement de l'ITRS, les ambigüités de son émergence : à la fois moyen d'établir un oligopole, mais aussi un oligopole fondé sur une dynamique d'exploration très élevée. Soit la cible oligopolistique étant atteinte, l'ITRS pouvait disparaitre, soit la capacité à suivre le rythme technologique est elle-même en péril. D'une certaine façon I'ITRS avait tendance à imposer un rythme qu'Intel n'arrivait plus à suivre. On voit aussi la montée d'une gouvernance très orientée sur le profit qui a pu diminuer la puissance d'action des R\&D internes d'Intel et qui a pu conduire les firmes à préférer un oligopole hyper stable plutôt qu'un oligopole dont la croissance est basée sur une très forte dynamique technique.

\section{5- Les autres organisations supports à la dynamique d'expertise}

PLM \&BW: L'exemple AENEAS, l'association pour laquelle Patrick Cogez travaille aujourd'hui, est intéressant pour apporter une autre perspective que celle de l'ITRS et de la loi de Moore. Deux aspects sont particulièrement intéressants, la question de l'inclusion dans le processus des organisation "clients " du semi-conducteur, et les enjeux sur la qualité des roadmap ainsi construites avec comme objectif de minimiser les fixations.

PC : AENEAS est une association industrielle qui promeut la R\&D et l'innovation dans les composants et systèmes électroniques pour renforcer la compétitivité européenne. Aujourd'hui on développe au niveau Européen un Agenda Stratégique de Recherche (Stratégic 
Research Agenda - SRA), qui vient nourrir le contenu des appels à projets de recherche européens dans ce domaine. Le partenariat européen public-privé ECSEL, ou certains programmes Eureka (Penta, Euripides) utilisent ainsi le SRA. Le SRA fournit d'abord un éclairage pour les financements. Mais évidemment il donne aussi des orientations pour la recherche. Nous essayons d'organiser un dialogue entre les fournisseurs de technologies et leurs utilisateurs, notamment dans les domaines qui sont les points forts de l'Europe comme le transport (automobile, mais également aéronautique, ferroviaire et maritime), la santé, l'énergie, l'industrie 4.0 et les smart cities. Donc nous essayons d'organiser des ponts entre les roadmaps produites par ces différentes communautés et la roadmap sur l'aspect technologique au niveau européen pour définir les besoins de recherche sur les dix ans qui viennent.

Par rapport à l'ITRS, qui se basait sur la loi de Moore dont on a dit qu'elle permettait jusqu'à un certain point d'ignorer les usages, là on essaie de comprendre et d'intégrer les besoins futurs des usagers et dans l'autre sens essayer de dire ce que la technique est capable de faire pour que les entreprises développant les applications puissent le prendre en compte. Le travail se fait à un horizon à 10 ans. Environ 250 experts sont mobilisés pour élaborer le SRA qui comporte 5 chapitres orientés application et 5 chapitres orientés technologie.

Disposer d'une roadmap qui oriente les financements vers des projets de recherche collaboratifs soulève bien sûr des questions: est-ce que les voies de recherche sont suffisamment couvertes ? Ou plus exactement, les voies absentes le sont-elles volontairement ou pas ? Les projets financés in fine couvrent-ils les voies de recherche ou se concentrent-ils sur certaines voies au détriment d'autres?

Nous avons travaillé aujourd'hui avec la théorie C-K à enrichir les roadmaps et à vérifier la répartition des financements. Nous avons fait une première expérience sur quelques chapitres. C'était tout à fait éclairant et convaincant et nous allons l'étendre à d'autres chapitres. Cela permet d'identifier des voies nouvelles et surtout nous avons constaté que ces voies étaient absentes parce qu'elles ne correspondaient pas aux compétences des personnes qui rédigeaient le chapitre. Par exemple, au sein du chapitre "digital life ", la question du rapport des gens au temps oblige à travailler avec des sociologues sur ce que peut apporter le gain de temps. Compétences qui n'étaient pas associées jusque-là à l'écriture de ces roadmaps.

$\mathrm{CL}$ : Au Japon, ce souci des compétences nécessaires pour écrire les roadmaps futures est aussi présent, en particulier au niveau de l'organisation patronale. II y a quelques années, le Semiconductor Industry Research Institute Japan, une organisation dirigée par un ancien de NEC et faisant l'interaction entre l'industrie des semi-conducteurs et le MITI, a rédigé des rapports sur la société numérique.

\section{En conclusion :}

CL : La loi de Moore, c'est un choix technique qui a été fait par Gordon Moore quand il était à Fairchild, dès 1965 et peut-être même dès 1963. Mais c'est aussi un outil de gestion. Moore et ses équipes ont utilisé la loi de Moore pour attribuer les ressources et gérer la conception des procédés et des produits à Fairchild et Intel. En ce sens, c'est une forme de planning technique. La loi de Moore est aussi un outil de marketing et de vente de produits. L'article de Gordon Moore, qui en 1965 a introduit l'idée du doublement du nombre de transistors 
tous les ans, était un texte de marketing destiné à convaincre les entreprises d'électronique à acheter des circuits intégrés. Enfin, la loi de Moore est un outil de discussion avec les fournisseurs et les utilisateurs. Dès la fin des années 1960, Moore est allé dans les congrès d'ingénieurs qui travaillaient en aval des semi-conducteurs pour leur expliquer les types de circuits qui allaient arriver et pour stimuler leur imagination et leur créativité sur les applications qu'ils pourraient développer sur la base des futures générations technologiques. C'était une façon d'attirer les utilisateurs, de les stimuler, de les faire réagir et de leur demander ce qui les intéresse vraiment.

PC : Cela attire les utilisateurs technologues et même jusqu'aux utilisateurs finaux - le slogan "Intel inside » reste surprenant: on essaie de convaincre un utilisateur d'acheter un objet complexe comme un ordinateur en vantant les qualités d'un composant qu'il ne verra jamais, le microprocesseur!

$\mathrm{CL}$ : Intel a longtemps eu des circuits intégrés moins bons que ceux de la concurrence, Motorola par exemple. La loi de Moore a convaincu les clients d'acheter les produits d'Intel parce que la promesse de vitesse d'évolution d'une génération à l'autre permettait de compenser les limites de performance de ses circuits intégrés par rapport à ceux de la concurrence. Intel ne vend pas des produits qui existent. II pousse les ingénieurs de compagnies clientes à penser comme si un produit qui n'existe pas encore existe déjà et du coup à concevoir des nouveaux dispositifs autour de ce produit. 